\title{
Brevundimonas vesicularis Causing Bilateral Pneumosepsis in an Immunocompetent Adult: A Case Report and Literature Review
}

\author{
Sarah N Stabler, Benjamin Mack, Grant McCormack, and Matthew P Cheng
}

\section{INTRODUCTION}

$B$ revundimonas vesicularis is an aerobic, nonfermenting gram-negative bacillus that produces oxidase and catalase. ${ }^{1}$ Previously known as Pseudomonas vesicularis, this organism was reclassified under the genus Brevundimonas on the basis of differentiating genotypic and phenotypic characteristics. ${ }^{1}$ It is commonly found in soil and water, is considered ubiquitous in the environment, and is a rare cause of infection in humans. ${ }^{1-4}$ Most case reports have described infection with Brevundimonas spp. in immunocompromised patients, although there are isolated reports of infection in immunocompetent patients. ${ }^{2-5}$ To the authors' knowledge, there are no published case reports to date describing $B$. vesicularis pneumonia in immunocompromised or immunocompetent patients.

\section{CASE REPORT}

A previously healthy adult patient (age early 40s) presented to the emergency department with a 4-day history of fever, cough, and shortness of breath.* On admission, the rectal temperature was $39.8^{\circ} \mathrm{C}$, heart rate 133 beats $/ \mathrm{min}$, blood pressure $137 / 84 \mathrm{~mm} \mathrm{Hg}$, oxygen saturation $91 \%$ on room air, and respiratory rate 26 breaths/min. The patient had originally resided in India, but denied any travel outside Canada during the previous year. The patient's HIV status was negative, there was no evidence of immunoglobulin deficiency, and there were no known sick contacts or recent interactions with the health care system.

Chest radiography on admission showed bilateral pulmonary infiltrates, larger on the right side than the left. The initial leukocyte count was $2.4 \times 10^{9} / \mathrm{L}$ (normal range $4-11 \times$

\footnotetext{
*Despite repeated attempts, the authors were unable to reach the patient to request consent for publication of this report. Therefore, personal details not pertinent to understanding of the case have been omitted to protect confidentiality.
}

$10^{9} / \mathrm{L}$ ), with a neutrophil count of $1.7 \times 10^{9} / \mathrm{L}$ (normal range $2-8 \times 10^{\%} / \mathrm{L}$ ) and eosinophils undetectable. Serum sodium was $121 \mathrm{mmol} / \mathrm{L}$ (normal range 135-145 mmol/L), potassium $3.1 \mathrm{mmol} / \mathrm{L}$ (normal range $3.5-5 \mathrm{mmol} / \mathrm{L}$ ), C-reactive protein $209 \mathrm{mg} / \mathrm{L}$ (normal range $<7.5 \mathrm{mg} / \mathrm{L}$ ), $\gamma$-glutamyl transpeptidase $466 \mathrm{U} / \mathrm{L}$ (normal range $<31 \mathrm{U} / \mathrm{L}$ ), and lactate dehydrogenase $531 \mathrm{U} / \mathrm{L}$ (normal range < $220 \mathrm{U} / \mathrm{L}$ ); all other laboratory results were within normal limits. Examination showed bilateral coarse crackles in both lung fields, but all other physical findings were unremarkable.

The patient was stabilized, and blood and urine samples were sent for culture and sensitivity testing; the patient was unable to expectorate sputum sample adequate for testing. Supplemental oxygen and antibiotic therapy, with IV administration of ceftriaxone and azithromycin, were initiated for a provisional diagnosis of community-acquired pneumonia. Despite appropriate empiric treatment, there were no signs of clinical improvement. After 3 days, the patient's respiratory system decompensated, with progressive hypoxemia and hypotension necessitating additional supplemental oxygen, vasopressor support, intubation, and mechanical ventilation.

At this time, culture results for blood and urine samples obtained at the time of presentation became available; all were negative. However, because of the patient's declining clinical status, azithromycin and ceftriaxone were discontinued, and IV therapy with piperacillin-tazobactam was started.

An endotracheal aspirate sent for culture and sensitivity testing at the time of intubation was found to be mucopurulent, and eventually grew Brevundimonas vesicularis. The results of additional testing (multiplex viral polymerase chain reaction assay, and antigen testing for Legionella pneumophilia and Mycoplasma pneumoniae) were negative. Subsequent quantitative culture of samples obtained during bronchoscopy yielded negative results. Acid-fast staining for Mycobacterium tuberculosis and immunofluorescent staining for Pneumocystis jirovecii 
(Fungi-Fluor kit for fungal detection, Polysciences, Inc, Warrington, Pennsylvania) were also negative. All additional culture results were negative.

Bronchoscopy performed $36 \mathrm{~h}$ after intubation showed inflamed airways, but the findings were otherwise unremarkable. Mucopurulent respiratory secretions were noted; no hemorrhage, obstruction, or tumours were visualized. Samples taken during bronchial washing showed no malignant cells and no fungal elements. Contrast-enhanced computed tomography of the chest showed widespread centrilobular pulmonary nodularity with ill-defined margins and ground-glass density. Confluent airspace consolidation was identified in the subpleural lower lobes and in the right middle lobe. Trace pleural effusions were noted.

The patient gradually began to improve, with extubation after 5 days of mechanical ventilation. A 14-day course of piperacillin-tazobactam was given, and the patient was eventually discharged home with no sequelae.

\section{Microbiology Results}

Gram staining of the endotracheal aspirate obtained on the day of intubation showed $2+$ polymorphonuclear cells, $2+$ monomorphonuclear cells, $1+$ squamous epithelial cells, $1+$ organisms suggestive of respiratory flora, and $1+$ yeast (quantitation per local laboratory protocol). After $48 \mathrm{~h}$ of incubation on sheep's blood agar and chocolate agar, there was heavy growth of grey colonies that were nonhemolytic and did not form satellites. Gram staining showed these organisms to be small gram-negative bacilli. The results of oxidase and porphyrin fluorescence tests were negative. The organism was identified by the API 20 NE identification system (bioMérieux) as B. vesicularis (profile 1440200, 98.3\% probability). ${ }^{6}$ Furthermore, the isolate tested positive for esculin hydrolysis, D-glucose assimilation, and D-maltose assimilation, results that strongly corroborated the identification and reliably differentiated the organism from B. diminuta? ${ }^{7}$

Susceptibility testing was performed with the Kirby-Bauer disk diffusion method, using an inoculum of $0.5 \mathrm{McF}$ arland standard on Mueller-Hinton agar and interpreted according to the performance standards of the Clinical and Laboratory Standards Institute (M100 document, 17th edition, Table 2B-1, "Zone diameter interpretive standards and equivalent minimal inhibitory concentration [MIC] breakpoints for Pseudomonas aeruginosa and non-Enterobacteriaceae"). ${ }^{8}$ The organism was reported as susceptible to the following antibiotics (zone diameters in parentheses): meropenem (42 mm), piperacillin $(45 \mathrm{~mm})$, gentamicin $(50 \mathrm{~mm})$, tobramycin (45 mm), and trimethoprim-sulfamethoxazole $(52 \mathrm{~mm}$ ); the organism was reported as resistant to the following antibiotics: ceftazidime $(0 \mathrm{~mm})$ and ciprofloxacin $(0 \mathrm{~mm})$.

\section{DISCUSSION}

To the authors' knowledge, this is the first reported case describing $B$. vesicularis as a causative pathogen for pneumonia.

From the literature search, we identified a total of 78 cases, from 21 case reports and 3 case series (where a case series was defined as $n \geq 4$ ), describing $B$. vesicularis as a causative pathogen..$^{2-5,9-28}$ There was high variability among the cases as to the site and severity of infection, the age and immune status of the patient, and the geographic location. However, most cases occurred in immunocompromised adults and were described as bacteremia or sepsis, with no focus of infection reported. 2,5,9-11,13-16,19,22-25,27 No previously reported cases of pneumonia with $B$. vesicularis as causative pathogen were identified.

Because of the high variability in antibiotic susceptibility patterns from the previously reported cases, we were unable to choose an empiric antibiotic with confidence. Brevundimonas vesicularis has often been described as susceptible to piperacillintazobactam, carbapenems, and aminoglycosides. ${ }^{2-4,10-15,17,20,22,24,25,27}$ However, resistance to these antibiotics has also been reported. ${ }^{4,11,14,15,17,20}$ We chose piperacillin-tazobactam, to avoid selective pressure on carbapenem-resistant organisms and because this drug has superior lung penetration relative to aminoglycosides. The reported susceptibility to third- and fourth-generation cephalosporins and fluoroquinolones is much less consistent, with the largest case series reporting $0 \%-86.4 \%$ of isolates susceptible to ciprofloxacin and 3\%-63.6\% of isolates susceptible to ceftazidime. ${ }^{2,27}$ There has also been a high degree of variability in the methods used for testing susceptibility in the reported cases, which makes the results difficult to interpret.

\section{CONCLUSION}

For the case reported here, we were unable to determine where the patient might have contracted infection with this bacterium or why it caused such a severe infection in a relatively young, healthy adult. Although infections with Brevundimonas spp. are uncommon, as revealed by our literature search, this case shows the potential for such an infection to cause bilateral pneumonia and septic shock in an immunocompetent adult. Given the high variability in antibiotic susceptibility that has been reported to date, consideration should be given to initiating empiric therapy with piperacillin-tazobactam or an antipseudomonal carbapenem in patients with known or suspected $B$. vesicularis infections.

\section{References}

1. Segers P, Vancanneyt M, Pot B, Torck U, Hoste B, Dewettinck D, et al. Classification of Pseudomonas diminuta Leifson and Hugh 1954 and Pseudomonas vesicularis Büsing, Döll, and Freytag 1953 in Brevundimonas gen. nov. as Brevundimonas diminuta comb. nov. and Brevundimonas vesicularis comb. nov., respectively. Int J Syst Bacteriol. 1994;44(3):499-510.

2. Lee MR, Huang YT, Liao CH, Chuang TY, Lin CK, Lee SW, et al. Bacteremia caused by Brevundimonas species at a tertiary care hospital in Taiwan, 2000-2010. Eur J Clin Microbiol Infect Dis. 2011;30(10):1185-91. 
3. Sofer Y, Zmira S, Amir J. Brevundimonas vesicularis septic arthritis in an immunocompetent child. Eur J Pediatr. 2007;166(1):77-8.

4. Yang ML, Chen YH, Chen TC, Lin WR, Lin CY, Lu PL. Case report: infective endocarditis caused by Brevundimonas vesicularis. BMC Infect Dis. 2006;6:179

5. Gilad J, Borer A, Peled N, Riesenberg K, Tager S, Appelbaum A, et al. Hospital-acquired Brevundimonas vesicularis septicaemia following open-heart surgery: case report and literature review. Scand J Infect Dis. 2000;32(1): 90-1.

6. Geiss HK, Piotrowski HD, Hingst V. Evaluation of API 20 NE in routine diagnostics of nonfermenting gram-negative rod-shaped bacteria. Zentralbl Bakteriol Mikrobiol Hyg A. 1985;259(1):35-42.

7. Pfaller MA, Richter SS, Funke G, Jorgensen JH, Landry ML, Carroll KC, et al., editors. Manual of clinical microbiology. 11th ed. Washington (DC): American Society of Microbiology; 2015.

8. Performance standards for antimicrobial susceptibility testing. 17th ed. Wayne (PA): Clinical and Laboratory Standards Institute; 2007.

9. Ben Haj Khalifa A, Bouzidi H, Sfar MT, Kheder M, Ayadi A. Bactériémie à Brevundimonas vesicularis chez un nourrisson atteint de la maladie de Pompe [Brevundimonas vesicularis bacteremia in an infant presenting with Pompe disease]. Med Mal Infect. 2012;42(8):370-1. Article in French.

10. Bhatawadekar SM, Sharma J. Brevundimonas vesicularis bacteremia: a rare case report in a female infant. Ind J Med Microbiol. 2011;29(4):420-2.

11. Calegari L, Gezuele E, Torres E, Carmona C. Botryomycosis caused by Pseudomonas vesicularis. Int J Dermatol. 1996;35(11):817-8.

12. Chi CY, Fung CP, Wong WW, Liu CY. Brevundimonas bacteremia: two case reports and literature review. Scand J Infect Dis. 2004;36(1):59-61.

13. Choi W, Lee C, Kim A, Choi JW, Seo S, Lee J, et al. CAPD peritonitis due to Brevundimonas vesicularis. Perit Dial Int. 2006;26(4):510-2.

14. Gupta PK, Appannanavar SB, Kaur H, Gupta V, Mohan B, Taneja N. Hospital acquired urinary tract infection by multidrug-resistant Brevundimonas vesicularis. Indian J Pathol Microbiol. 2014;57(3):486-8.

15. Karadag N, Karagol BS, Kundak AA, Dursun A, Okumus N, Tanir G, et al. Spectrum of Brevundimonas vesicularis infections in neonatal period: a case series at a tertiary referral center. Infection. 2012;40(5):509-15.

16. Lin $\mathrm{CY}$, Chen YH. Bacteremia due to Brevundimonas vesicularis [letter]. J Microbiol Immunol Infect. 2013;46(2):143.

17. Mondello P, Ferrari L, Carnevale G. Nosocomial Brevundimonas vesicularis meningitis. Infez Med. 2006;14(4):235-7.

18. Niedermeier DM, Frei-Lahr DA, Hall PD. Treatment of acute myeloid leukemia during the second and third trimesters of pregnancy. Pharmacotherapy. 2005;25(8):1134-40.

19. Oberhelman RA, Humbert JR, Santorelli FW. Pseudomonas vesicularis causing bacteremia in a child with sickle cell anemia. South Med J. 1994; 87(8):821-2.

20. Panasiti V, Devirgiliis V, Mancini M, Curzio M, Rossi M, Fioriti D, et al. A cutaneous infection caused by Brevundimonas vesicularis: a case report [letter]. Int J Immunopathol Pharmacol. 2008;21(2):457-61.

21. Pelletier JS, Ide T, Yoo SH. Brevundimonas vesicularis keratitis after laser in situ keratomileusis. J Cataract Refract Surg. 2010;36(2):340-3.
22. Planes AM, Ramirez A, Fernandez F, Capdevila JA, Tolosa C. Pseudomonas vesicularis bacteraemia. Infection. 1992;20(6):367-8.

23. Sève S, Mohammedi I, Martin O, Argaud L, Michallet AA, Berchiche C, et al. Bactériémie à Brevundimonas vesicularis chez une patiente en aplasie [Brevundimona vesicularis bacteremia in a patient in aplasia]. Presse Med. 2004;33(3):172. Article in French.

24. Shang ST, Chiu SK, Chan MC, Wang NC, Yang YS, Lin JC, et al. Invasive Brevundimonas vesicularis bacteremia: two case reports and review of the literature. J Microbiol Immunol Infect. 2012;45(6):468-72.

25. Vanholder R, Vanhaecke E, Ringoir S. Pseudomonas septicemia due to deficient disinfectant mixing during reuse. Int J Artif Organs. 1992;15(1): 19-24.

26. Yoo SH, Kim MJ, Roh KH, Kim SH, Park DW, Sohn JW, et al. Liver abscess caused by Brevundimonas vesicularis in an immunocompetent patient. J Med Microbiol. 2012;61(10):1476-9.

27. Zhang CC, Hsu HJ, Li CM. Brevundimonas vesicularis bacteremia resistant to trimethoprim-sulfamethoxazole and ceftazidime in a tertiary hospital in southern Taiwan. J Microbiol Immunol Infect. 2012;45(6):448-52.

28. Karadag N, Karagol BS, Dursun A, Okumus N, Tanir G, Zenciroglu A. A premature neonate with early-onset neonatal sepsis owing to Brevundimonas vesicularis complicated by persistent meningitis and lymphadenopathy. Paediatr Int Child Health. 2012;32(4):239-41.

Sarah N Stabler, BSc(Pharm), ACPR, PharmD, is with the Department of Critical Care and the Department of Pharmacy Services, Surrey Memorial Hospital, Surrey, British Columbia.

Benjamin Mack, MD, FRCPC, is with the Department of Laboratory Medicine and Pathology, Surrey Memorial Hospital, Surrey, British Columbia.

Grant McCormack, MD, FRCPC, is with the Department of Critical Care, Surrey Memorial Hospital, Surrey, British Columbia.

Matthew P Cheng, MD, FRCPC, is with the Division of Infectious Diseases and the Department of Medical Microbiology, McGill University Health Centre, Montréal, Quebec.

Competing interests: None declared.

\section{Address correspondence to:}

Dr Sarah N Stabler

Surrey Memorial Hospital

13750 96th Avenue

Surrey BC V3V 1 Z2

e-mail: Sarah.Stabler@fraserhealth.ca

Funding: None received. 